\title{
A Method to Recover Counting Distributions from Their Characteristic Functions
}

John A. Gubner, Member, IEEE, and Majeed M. Hayat, Member, IEEE

\begin{abstract}
A method that does not use numerical integration is presented for approximating the cumulative distribution of integer-valued random variables from their characteristic functions. Bounds on the approximation error are also given. The method is then used to compute photomultiplier counting distributions.
\end{abstract}

\section{INTRODUCTION}

$\mathbf{T}$ THE design of systems with photonic components often requires the calculation of false-alarm and detection probabilities of the form $P(X \geq n)$ or $P(X \leq n)$ where $X$ is an integer-valued random variable. Examples include optical communication systems [3], optical neural networks [4], and photomultipliers [5]. In these cases, one typically has a simple formula for the characteristic function of $X \varphi(u):=E\left[e^{j u X}\right]$, but not for $P(X \geq n)$ and $P(X \leq n)$.

The most obvious method to recover these probabilities from $\varphi$, which is $2 \pi$ periodic, is to recall that $P(X=k)$ is the $k$ th Fourier coefficient of $\varphi$. Then note that $P(X \leq n)=$ $\sum_{k=-\infty}^{n} P(X=k)$ and $P(X \geq n)=\sum_{k=n}^{\infty} P(X=k)$. The two sources of error here are the numerical integration error in computing the Fourier coefficients $P(X=k)$ and the error incurred by truncating the infinite sums. A less obvious method involves numerical contour integration in the complex plane using saddle-point techniques [5], [6].

In this paper we present a new method for approximating $P(X \geq n)$ and $P(X \leq n)$ using $\varphi$. Our method, given in the theorem below and proved in Section II, includes simple error bounds and does not involve numerical integration. Also, the method can take advantage of the fast Fourier transform if several probabilities are needed at the same time, e.g., for graphical or optimization purposes.

Theorem: If $X$ is an integer-valued random variable with characteristic function $\varphi$, then

$$
P(X \geq n)=\lim _{L \rightarrow \infty} \hat{p_{L}}(n)
$$

Manuscript received August 14, 1995. The associate editor coordinating the review of this letter and approving it for publication was Prof. J. M. F. Moura

This work was supported by the Air Force Office of Scientific Research under Grant F49620-92-J-0305. This work was presented at the Conference on Applied Probability in Engineering, Computer and Communication Sciences, Paris, France, June 16-18, 1993.

J. A. Gubner is with the Department of Electrical and Computer Engineering, University of Wisconsin, Madison, WI 53706 USA (e-mail: gubner@engr.wisc.edu)

M. M. Hayat was with the Department of Electrical and Computer Engineering, University of Wisconsin, Madison, WI 53706 USA. He is now with the Electro-Optics Graduate Program, University of Dayton, Dayton, OH 45469 USA.

Publisher Item Identifier S 1070-9908(96)04932-2. where

$$
\hat{p_{L}}(n):=\frac{1}{2 L} \sum_{k=0}^{2 L-1} \hat{\beta_{L}}(k) \varphi(k \pi / L) e^{-j n k \pi / L}
$$

$$
\hat{\beta_{L}}(k)= \begin{cases}L, & k \text { a multiple }{ }^{1} \text { of } 2 L \\ 1-j \cot \left(\frac{k \pi}{2 L}\right), & k \text { odd } \\ 0, & \text { otherwise. }\end{cases}
$$

Furthermore, we have the error bounds

$$
\hat{p_{L}}(n)-P(X \geq n) \leq P(X<-L+n)
$$

and

$$
\hat{p_{L}}(n)-P(X \geq n) \geq-P(X \geq L+n) \text {. }
$$

Remarks:

1) By defining $Z=-X$ and noting that $P(X \leq n)=$ $P(Z \geq-n)$, the theorem easily implies that (since $P(X \leq n)$ is real, and since the characteristic function of $Z$ is the complex conjugate of $\varphi$ )

$P(X \leq n)=\lim _{L \rightarrow \infty} \frac{1}{2 L} \sum_{k=0}^{2 L-1} \hat{\beta_{L}}(-k) \varphi(k \pi / L) e^{-j n k \pi / L}$.

2) Equation (1) can be interpreted as a sampling theorem for the periodic "waveform" $\varphi$ with discrete "spectrum" $P(X=n)$. If $X$ is a bounded random variable, i.e., the spectrum is bandlimited, then $P(X \geq n)=\hat{p_{L}}(n)$ for sufficiently large but finite $L$; this can be seen by noting that the error bound in (3) is zero if $-L+n$ is smaller than the minimum possible value of $X$; similarly, the bound in (4) is zero if $L+n$ is greater than the maximum possible value of $X$. For unbounded random variables, $P(X \geq n)$ does not exactly equal $\hat{p_{L}}(n)$ for finite $L$ due to aliasing, and the bounds (3)-(4) quantify the aliasing error.

3) As shown in the proof of the theorem, $\hat{\beta}_{L}$ is the discrete Fourier transform (DFT) of a discrete-time periodic pulse train.

4) Formulas analogous to (1) have been derived for random variables with a continuous cumulative distribution in [1] and [2].

\footnotetext{
${ }^{\mathrm{l}}$ Since $k=0$ is a multiple of $2 L, \hat{\beta_{L}}(0)=L$. See also $(10)$.
} 


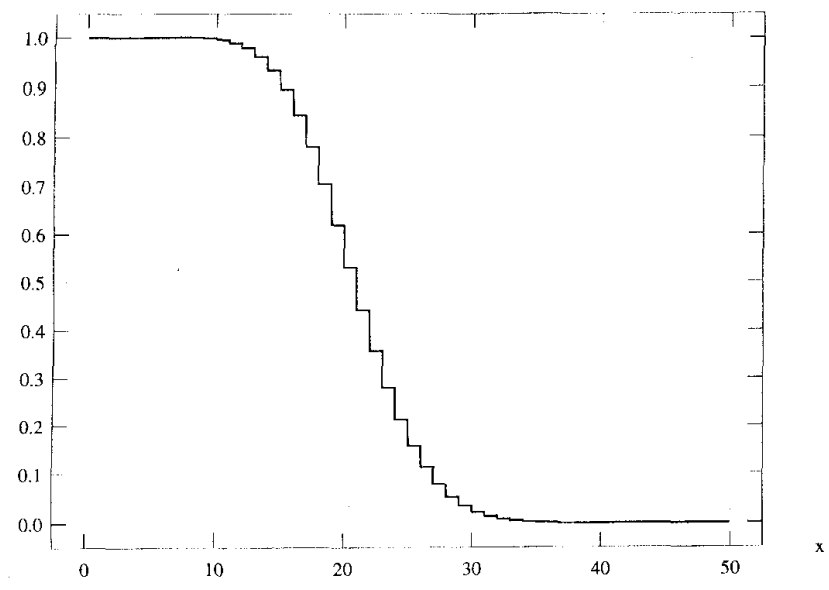

Fig. 1. $P(X \geq x)$ approximated using $\hat{p}_{64}(n)$.

In applications, one is generally interested in a finite range of $n$, and so one can take $L$ finite and then use a fast Fourier transform to compute the approximations $\hat{p_{L}}(n)$ all at once. If the moment-generating function of $X$ exists, the Chernoff bound can be used to give numerical bounds on the right-hand sides of (3) and (4). Note that if $X$ is nonnegative, the upper bound in (3) is zero for $n \leq L$, and the worst-case lower bound in (4) occurs at $n=0$.

Example 1: Let $X$ be a Poisson random variable with mean $\Lambda$. Then its moment-generating function is given by $E\left[e^{s X}\right]=\exp \left[\Lambda\left(e^{s}-1\right)\right]$. To bound the right-hand side of (4), note that for $n \geq 0$

$$
\begin{aligned}
P(X \geq L+n) & \leq P(X \geq L) \\
& =P(s X \geq s L), \quad \text { for } s>0 \\
& \leq e^{-s L} E\left[e^{s X}\right], \quad \text { by Markov's ineq. } \\
& =\exp \left[\Lambda\left(e^{s}-1\right)-s L\right] .
\end{aligned}
$$

The bound in (6) is minimized by taking $s=\ln (L / \Lambda)$. We now take $\Lambda=20$ and $L=64$. With $s=\ln (64 / 20)$, the bound in (6) is less than $7 \times 10^{-14}$. Using $\hat{p}_{64}$ to approximate $P(X \geq x)$, we obtain the graph shown in Fig. 1 .

Example 2: Consider a photomultiplier with a single stage of multiplication. Assume that the number of secondary electrons ejected by a single primary electron is Poisson with probability-generating function $g(z)=e^{G(z-1)} ; G$ is called the gain. Let the number of primary electrons have a negative binomial distribution with probability-generating function $f(z)=[(1-v) /(1-v z)]^{M}$, where $v:=N_{p} /\left(N_{p}+M\right) ; N_{p}$ is the expected number of primaries, and $M$ is the number of degrees of freedom [5, pp. 297-298]. Let $X$ represent the total number of secondary electrons produced by all of the primaries. Then the moment-generating function of $X$ is $f\left(g\left(e^{s}\right)\right)$, and $\varphi(u)=f\left(g\left(e^{j u}\right)\right)$. To compare (5) with the exact results in [5], we took $N_{p}=18, G=72$, and $M=5$, and then considered the approximation of $P(X<n)$ for $n=250,500,750,1000,1500$, and 2000. Since $P(X<$ $n)=P(-X \geq-(n-1))$, and $-X$ is a nonpositive random variable, the relevant error bound comes from (3), and proceeding as in the derivation of (6), we have

$$
P(X>L+(n-1)) \leq e^{-s(L+n-1)} f\left(g\left(e^{s}\right)\right) .
$$

For the calculations we are considering, the worst-case bound occurs at $n=250$, and the bound improves as $n$ increases. Some caution is needed in minimizing the bound in (7); from the definition of $f(z)$, we must restrict $|z|<1 / v$, and this implies $0<s<\ln (1-(\ln v) / G)$. The value of $s$ that minimizes (7) and the corresponding value of the bound can then be found by plotting the bound as a function of $s$ in the above range. For $n=250$ and $L=6000$, the bound in (7) is less than $8 \times 10^{-5}$ when $s=0.00262$. For $n=2000$ and $L$ still equal to 6000 , the bound in (7) is less than $7 \times 10^{-7}$ when $s=0.00278$. The approximate probabilities are, respectively, $0.01502,0.08133,0.20906,0.36937,0.672100 .860256$, which agree to the number of digits shown with column labeled "Exact" in [5, p. 315, Table VII].

\section{Proof OF THE THeOREM}

First note that since the right-hand sides of (3)-(4) go to zero as $L \rightarrow \infty$, the convergence in (1) is immediate once (3)-(4) is established.

Fix any integer $n$, and set $Y=X-n$. Then $P(X \geq n)=$ $P(Y \geq 0)$, and the characteristic function of $Y$ is $\varphi(u) e^{-j n u}$. Let $L$ be any positive integer, and define the discrete-time periodic pulse train $\beta_{L}$ with period $2 L$ by specifying its values on $\{0, \cdots, 2 L-1\}$ to be $\beta_{L}(m)=1$ for $m=0, \cdots, L-1$ and $\beta_{L}(m)=0$ for $m=L, \cdots, 2 L-1$. We show below that

$$
\hat{p_{L}}(n)=E\left[\beta_{L}(Y)\right] \text {. }
$$

Hence, the theorem will be proved if we can show that the difference

$$
E\left[\beta_{L}(Y)\right]-P(Y \geq 0)
$$

is upper bounded by the right-hand side of (3) and lower bounded by the right-hand side of (4).

We begin by proving (8). Let $\hat{\beta}_{L}$ denote the DFT of $\beta_{L}$. Then

$$
\hat{\beta_{L}}(k):=\sum_{m=0}^{2 L-1} \beta_{L}(m) e^{-j k m 2 \pi /(2 L)}=\sum_{m=0}^{L-1} e^{-j k m \pi / L} .
$$

This last sum is clearly given by (2) if we use the identity $2 /\left(1-e^{-j \theta}\right)=1-j \cot (\theta / 2)$. By the DFT inversion formula

$$
\beta_{L}(m)=\frac{1}{2 L} \sum_{k=0}^{2 L-1} \hat{\beta_{L}}(k) e^{j k m 2 \pi /(2 L)}
$$

and, hence, $E\left[\beta_{L}(Y)\right]=\hat{p_{L}}(n)$, which is exactly (8).

To conclude the proof, we show that (9) satisfies the error bounds. For any set $A$, let $I_{A}(m)=1$ for $m \in A$ and $I_{A}(m)=0$ otherwise. Observe that for all $m$

$$
\beta_{L}(m) \leq I_{[0, \infty)}(m)+I_{(-\infty,-L)}(m)
$$

and

$$
\beta_{L}(m) \geq I_{[0, L)}(m)=I_{[0, \infty)}(m)-I_{[L, \infty)}(m) .
$$


IEEE SIGNAL PROCESSING LETTERS, VOL. 3, NO. 6, JUNE 1996

Since $E\left[I_{[0, \infty)}(Y)\right]=P(Y \geq 0)$, the above inequalities easily imply that $(9)$ is upper bounded by $P(Y<-L)=P(X<$ $-L+n)$ and lower bounded by $-P(Y \geq L)=-P(X \geq$ $L+n$ ), thus proving (3)-(4).

\section{REFERENCES}

[1] N. C. Beaulieu, "An infinite series for the computation of the complementary probability distribution function of a sum of independent random variables and its application to the sum of Rayleigh random variables," IEEE Trans. Commun., vol. 38, pp. 1463-1474, 1990.
[2] J. A. Gubner, "Computation of shot-noise probability distributions and densities," SIAM J. Sci. Comput., vol. 17, no. 3, May 1996.

[3] M. M. Hayat, B. E. A. Saleh, and J. A. Gubner, "Bit-error rates for optical receivers using avalanche photodiodes with dead space," IEEE Trans. Commun., vol. 43, no. 1, pp. 99-106, Jan. 1995.

[4] - "Shot-noise-limited performance of optical neural networks," IEEE Trans. Neural Networks, vol. 7, no. 3, May 1996.

[5] C. W. Helstrom and S. O. Rice, "Computation of counting distributions arising from a single-stage multiplicative process," J. Comput. Phys. vol. 54, pp. 289-324, May 1984

[6] C. W. Helstrom, "Computation of photoelectron counting distributions by numerical contour integration," J. Opt. Soc. Amer. A, vol. 2, pp. 674-682, May 1985. 